\title{
Renovação de adutoras: uma abordage
ponto de vista da eficiência energética
}

\section{Renovation of water mains: an approach from the point of view of energy efficiency}

\author{
Data de entrada: \\ 28/05/2019 \\ - Data de aprovação: \\ $10 / 07 / 2019$
}

André Luís Sotero Salustiano Martim ${ }^{1 *}$ | Lucas Buonanno Perini ${ }^{1}$ | Daniela Bonazzi Sodek ${ }^{1}$ | Elias Sebastião Amaral Tasca' | José Gilberto Dalfré Filho'

DOI: https://doi.org/10.36659/dae.2020.076

ORCID ID

Salustiano Martim ALS (D) https://orcid.org/0000-0002-3578-0719

Perini LB (ID) https://orcid.org/0000-0002-2971-5574
Sodek DB (1) https://orcid.org/0000-0002-9639-9444

Tasca ESA https://orcid.org/0000-0001-6197-9329

Dalfré Filho JG (D) https://orcid.org/0000-0001-8105-6177

\section{Resumo}

Frequentes rompimentos devido a transitórios hidráulicos, excesso de consumo de energia e material antiquado da tubulação tornaram necessária a substituição de uma adutora. Os tubos de cimento-amianto DN $125 \mathrm{~mm}$ foram substituídos por tubos de PVC DEFOFO DN $300 \mathrm{~mm}$. Neste trabalho, investiga-se a relação entre consumo de energia e diâmetro, e os métodos de Bresse e de Prevedello para escolha de diâmetros econômicos são discutidos. A solução adotada pela concessionária gerou $23,8 \%$ de economia de energia. A avaliação do diâmetro ótimo para a adutora a partir dos dados fornecidos pela concessionária resultou em $D=210 \mathrm{~mm}$. A fórmula de Bresse resultou em $D=179 \mathrm{~mm}$. A aplicação do método de Prevedello resultou em $D=194 \mathrm{~mm}$. Ficou evidenciada a necessidade da aplicação de metodologias adequadas para a otimização de diâmetros de adutoras. Palavras-chave: Dimensionamento de Adutoras, Eficiência Energética, Diâmetro Ótimo.

\section{Abstract}

Frequent bursts because of hydraulic transients, excessive energy consumption and outdated pipeline material were the reasons for the replacement of a water main. The DN $125 \mathrm{~mm}$ asbestos pipes were replaced by DN $300 \mathrm{~mm}$ PVCDEFOFO pipes. In this paper, the relationship between energy consumption and pipe diameter is investigated, and the Bresse's and Prevedello's methods for economical diameters are discussed. The solution adopted by the water company generated a decrease in energy consumption of $23,8 \%$. The evaluation of the optimum diameter based on the data provided by the water company resulted in $D=210 \mathrm{~mm}$. The use of the Bresse equation resulted in $D=179 \mathrm{~mm}$. The application of the Prevedello method resulted in $D=194 \mathrm{~mm}$. The necessity of the application of proper methodologies for the achievement of diameter optimization is evinced.

Keywords: Water Mains Sizing, Energy Efficiency, Optimum Diameter.

\footnotetext{
${ }^{1}$ Universidade Estadual de Campinas - Campinas - São Paulo - Brasil.

* Autor correspondente: soteroafec.unicamp.br.
} 


\section{INTRODUÇÃO}

Em um sistema de abastecimento de água, a eletricidade é essencial para os processos de captação, tratamento e distribuição. A economia de água, que está intimamente ligada a aspectos ambientais, está também relacionada com a economia de energia elétrica. Estratégias para melhorar a eficiência energética dos sistemas de abastecimento têm importante apelo econômico (RAMOS et al., 2011).

A energia consumida por uma estação de bombeamento depende de diversos fatores: potência e eficiência da bomba, diâmetro e comprimento da adutora, fator de atrito do material dos tubos, vazão requerida e desnível geométrico (PLAPPALLY; LIENHARD, 2012). Em um projeto de adutora, a vazão e o desnível geométrico são conhecidos. No entanto, o diâmetro da tubulação e a potência da bomba são indeterminados e interdependentes.

\subsection{Transitórios hidráulicos em adutoras de recalque}

Adutoras de recalque estão sujeitas a frequentes sub-pressões e sobre-pressões transitórias advindas dos procedimentos de ligamento e desligamento dos sistemas de bombeamento. De acordo com a Eq. 1, Equação de Joukowsky, quanto maior a velocidade do escoamento e, em consequência, sua variação durante uma manobra $\Delta \mathrm{V}$, maior será a intensidade da variação da carga hidráulica transitória $\Delta \mathrm{H}$ (Tullis, 1989). $\mathrm{O}$ uso de diâmetros maiores resulta em menor velocidade da onda de sobre-pressão, amenizando a intensidade de $\Delta \mathrm{H}$.

$$
\Delta H=-\frac{a \Delta V}{g}
$$

A repetição de pressões transitórias causa a diminuição da resistência do material dos tubos devido à fadiga. Dessa forma, com o passar do tempo, a possibilidade de rupturas aumenta. Dentre as possíveis soluções para esse problema, pode-se citar o uso de dispositivos de proteção, prolongamento do período de recalque, que consequentemente diminuiria a vazão, ou ainda a alteração das características da adutora. Nesse último caso, a alteração do diâmetro, por exemplo, também apresenta a vantagem de diminuir o consumo de energia.

A análise das pressões extremas transitórias pode ser feita com o auxílio de softwares de simulação. Esses softwares podem também ser usados para a obtenção de diâmetros. Para esse fim, durante o período de testes do modelo hidráulico, é necessário o estabelecimento de velocidades de escoamento, respeitando-se as normas específicas para cada aplicação. Por meio da análise dos resultados fornecidos pelo software, vazão, perda de carga e pressões, para diferentes velocidades e diâmetros de tubo do material escolhido, pode-se encontrar um valor para o diâmetro dos dutos.

\subsection{Diâmetros otimizados para adutoras}

Em relação ao dimensionamento de adutoras, deve-se atentar para o fato de que uma solução hidraulicamente viável não necessariamente representa uma solução economicamente viável. Para uma mesma vazão e altura geométrica, existem inúmeras combinações de diâmetro e potência possíveis, sendo que quanto menor o diâmetro maior será a perda de carga e, consequentemente, maior será a potência exigida.

Muitos trabalhos foram desenvolvidos com foco na eficiência energética de sistemas de abastecimento de água. Ramos et al. (2011) apresentam três soluções para a otimização de sistemas de abastecimento de água: implantação de turbinas em pontos onde a redução de pressão é necessária, otimização no funcionamento das bombas de forma a ocorrer durante o período de tarifas 
econômicas de acordo com um modelo de tarifa horo-sazonal e implantação de energia eólica combinada com a energia convencional. Vilanova e Balestieri (2014) apresentam uma revisão de trabalhos realizados nessa área e concluem que a maioria dos projetos de sistemas de abastecimento de água se preocupa apenas com o custo do investimento, deixando de lado o custo operacional. Pode-se economizar, segundo esses autores, entre $5 \%$ e $20 \%$ de energia com o aumento dos diâmetros das tubulações. No entanto, a sustentabilidade ainda não é foco dos projetos de sistemas de abastecimento.

A fórmula de Bresse ainda é uma das mais utilizadas para o dimensionamento do diâmetro econômico de adutoras, apesar de simples e aproximada. A expressão parte das seguintes hipóteses: sistema funcionando $24 \mathrm{~h}$ por dia, conjunto motor-bomba de baixa potência, custo da tubulação diretamente proporcional ao diâmetro e ao comprimento e custo do conjunto motor-bomba diretamente proporcional à potência instalada (PORTO, 2006). Para o cálculo do custo unitário da tubulação, considera-se o gasto anual de $1 \mathrm{~m}$ de comprimento de um conduto de $1 \mathrm{~m}$ de diâmetro, incluindo despesas de amortização e conservação. Para o custo unitário do conjunto motor bomba, é considerado o custo anual de operação do conjunto motor-bomba por unidade de potência, incluindo despesas de operação e manutenção. $O$ custo total do sistema é determinado pela soma dos dois custos, de acordo com a Eq. 2.

$$
C_{t}=p_{1} L D+p_{2} \text { Pot }
$$

$\mathrm{Na}$ Eq.2, Ct é o custo total, p1 é o custo unitário anual da tubulação, Lé o comprimento da tubulação, $D$ é o diâmetro da tubulação, $p_{2}$ é o custo unitário anual de operação do conjunto motor-bomba e Pot é a potência do conjunto motor-bomba. Substituindo-se a equação da potência da bomba na Eq.2, obtém-se a Eq.3.
$C_{t}=p_{1} L D+p_{2} \frac{9,8 Q\left(H_{g}+0,0827 \frac{f L}{D^{5}} Q^{2}\right)}{\eta \eta_{m}}$

$\mathrm{Na}$ Eq.3, Q é a vazão de bombeamento, $\mathrm{H}_{\mathrm{g}}$ é a altura geométrica, fé o fator de atrito, L é o comprimento da tubulação, D é o diâmetro da tubulação, $\eta$ é o coeficiente de rendimento global da bomba e $\eta_{m}$ é o rendimento global do motor. $O$ custo mínimo total é obtido quando se iguala a zero a derivada primeira da Eq.3 em relação ao diâmetro, de onde se encontra a Eq.4 (fórmula de Bresse).

$D=\sqrt[6]{\frac{4,05 f}{\eta \eta_{m}} \frac{p_{1}}{p_{2}}} \sqrt{Q}$

Fazendo $\sqrt[6]{\frac{4,05 f}{\eta \eta_{m}} \frac{p_{1}}{p_{2}}}=K$, pode-se reescrever a Eq.4 conforme apresentado pela Eq. 5.

$D=K \sqrt{Q}$

Os valores de $\mathrm{K}$ variam com os custos regionais, entre outros fatores. Existe divergência entre autores com relação à faixa de valores de $\mathrm{K}$. No entanto, em geral, adota-se K entre 0,7 e 1,3. Porto (2006) recomenda a utilização da expressão de Bresse para adutoras de pequenos diâmetros (até 6 polegadas) e para diâmetros maiores recomenda apenas para a fase de pré-dimensionamento. Considerando-se a equação da continuidade na Eq.5, adotar o valor de $\mathrm{K}$ equivale a adotar uma velocidade econômica, como apresentado na Eq.6. Apesar de também haver diferença entre autores, normalmente a velocidade econômica é adotada entre 0,6 e $3,0 \mathrm{~m} / \mathrm{s}$.

$v=\frac{4}{\pi K^{2}}$

Apesar de muito utilizada, a fórmula de Bresse é frequentemente discutida por não considerar o 
regime do escoamento, limitar-se apenas à condução de água, considerar operação contínua da estação de bombeamento e estabelecer relação linear entre o diâmetro e o custo da tubulação. Ao longo dos anos, foram desenvolvidas outras metodologias variando as hipóteses iniciais adotadas por Bresse, principalmente quanto à relação linear entre custo e diâmetro, tempo de funcionamento e fatores que fazem parte do custo do investimento e do custo operacional.

Teles (1967) apresenta um método para o cálculo do diâmetro econômico de tubos de ferro fundido no qual a perda de carga é calculada por meio da equação empírica de Hazen-Williams. $O$ custo da energia é considerado diretamente proporcional à potência da bomba, o custo do tubo e da mão de obra é considerado proporcional ao peso do tubo. $O$ peso e o diâmetro se relacionam por meio de uma equação cúbica. Esse autor considera também que a variação do custo do conjunto motor-bomba tem pouca influência sobre o investimento total para a casa de bombeamento e pode ser desconsiderado.

Magnani (1968) também considera apenas o custo dos tubos na parcela de investimento. Similarmente a Bresse, a variação do custo da tubulação é considerada linear com o diâmetro e comprimento da tubulação. A perda de carga é calculada por meio da equação empírica de Hazen-Williams. O custo do consumo energético é considerado proporcional à potência do conjunto motor-bomba, porém são apresentadas duas abordagens: uma para bombas de rotação fixa, que considera o número de horas de funcionamento por dia, e outra para bombas de rotação variável, funcionando continuamente. Magnani (1968) considera um crescimento geométrico das vazões médias anuais durante um período de projeto de 20 anos.

Richter (1983) considera a variação do custo da tubulação com o diâmetro como sendo uma função exponencial $C=K D^{1,5}$. $O$ valor de $K$ é referen- te ao custo da tubulação por metro linear e é obtido por meio de um ábaco. Similarmente a Teles (1967) e Magnani (1968), esse autor desconsidera os custos das instalações de bombeamento e assentamento da adutora, pois considera pequena a variação com a alteração do diâmetro da tubulação. Dessa forma, a parcela do custo total do investimento refere-se apenas ao custo dos tubos. Para a composição do custo operacional, considera-se o trabalho necessário para elevação de $1 \mathrm{~m}^{3}$ de água a uma altura igual à perda de carga unitária.

Prevedello (2000) propõe um método para determinar o diâmetro econômico da tubulação de recalque levando em conta a natureza do líquido, o material dos tubos e o regime de escoamento. Em suas hipóteses, Prevedello (2000) considera os custos da canalização (incluindo a instalação) e o custo da operação da bomba. Porém, diferentemente de Bresse, não considera que o sistema opera continuamente ou que a variação do custo da tubulação seja linear.

$$
C_{\text {anual }}=\mathrm{p} 100 D^{\alpha} L \mathrm{r}+\mathrm{ns} \text { P } 365
$$

$\mathrm{Na}$ Eq.7, p é o custo da tubulação por $\mathrm{cm}$ de diâmetro e $\mathrm{m}$ de comprimento $(\mathrm{R} \$ / \mathrm{cm} / \mathrm{m})$, $D$ é o diâmetro da canalização $(\mathrm{m})$, $\alpha$ é o expoente que expressa a não linearidade entre o custo da canalização e o diâmetro, $L$ é o comprimento da canalização (m), r é a taxa de amortização da canalização, $P$ é a potência do conjunto motorbomba (W), $n$ é o número de horas de funcionamento diário do conjunto (h/dia) e s é o custo do kWh (R\$/kWh). Prevedello (2000) não considera perdas de carga localizadas e utiliza a fórmula universal para o cálculo da perda de carga distribuída, obtendo a Eq.8.

$$
C_{\text {anual }}=p 100 D^{\alpha} L r+n s 365 \frac{0,00981 \gamma Q^{3} f L 16}{\eta \pi^{2} D^{5} 2 g}
$$


A condição de mínimo custo é obtida igualandose a primeira derivada da Eq.8 a zero e desta forma a Eq.9 é obtida. Segundo Prevedello (2000), a segunda derivada da mesma equação deve ser maior do que zero. Essa condição será sempre assegurada, pois o valor do expoente $\alpha$ é sempre maior do que zero, uma vez que o custo da tubulação aumenta com o aumento do diâmetro. $O$ fator de atrito apresentado na Eq.9, em que $\alpha>0$, pode ser considerado como fator de atrito econômico. Escrevendo a Eq.9 de forma simplificada, tem-se a Eq.10.

$f=\frac{\eta \pi^{2} g p 100 r \alpha D^{(5+\alpha)}}{Q^{3} \gamma 40 \eta 0,0098 s 365}$

$f=f_{a} D^{(5+\alpha)}$

Igualando-se o fator de atrito conhecido pela equação de Colebrook-White, obtém-se a Eq.11 para o cálculo do diâmetro econômico. Na Eq.11, $k$ é a rugosidade absoluta do material $(\mathrm{m})$ e $v$ é a viscosidade do fluido ( $\mathrm{m} 2 / \mathrm{s})$.

$$
D=\frac{0,25^{\left(\frac{1}{5+\alpha}\right)}}{\left\{f_{a}\left[\log \left(\frac{k}{3,7 D}+\frac{2,15 v}{V D\left[f_{a} D^{(5+\alpha)}\right]}\right)\right]^{2,5}\right\}^{\left(\frac{1}{5+\alpha}\right)}}
$$

Para aplicar a Eq.11, é necessário usar um método iterativo, visto que a incógnita $D$ está em ambos os membros da expressão. No entanto, o método converge após poucas iterações.

Outros trabalhos foram publicados apresentando métodos semelhantes ao método de Prevedello (2000), cada um com sua particularidade. Por exemplo, Robaina et al. (2004) apresentam uma metodologia muito similar para tubulação de recalque para irrigação com base nos custos da tubulação, do conjunto motor-bomba e custo de energia consumida. A metodologia também utiliza a fórmula universal para a determinação da perda de carga e a equação de Colebrook-White para o cálculo do fator de atrito. $O$ preço da tubulação foi determinado por unidade de peso e por metro de tubo. Nesse caso, o material era aço soldado por ser comumente utilizado na região de Santa-Maria-RS. O preço do conjunto motorbomba foi determinado por unidade de potência. Essa metodologia também considera a operação da bomba intermitente e o diâmetro econômico é determinado por meio de método iterativo.

\subsection{0 problema do uso de cimento-amianto para tubulações}

De acordo com a International Agency for Research on Cancer (IARC), existem evidências suficientes para afirmar que todas as formas de cimentoamianto são cancerígenas. As fibras de cimentoamianto podem ser inaladas quando liberadas no ar durante a manutenção de tubulações (BOWEN et al., 2017). Muitos trabalhos foram desenvolvidos na investigação da relação entre a ingestão de cimento-amianto através da água e a incidência de câncer. Porém não existem evidências suficientes para confirmar essa hipótese IARC (2012).

Além da toxidade, tubulações de cimento-amianto são bastante suscetíveis a falhas. Mackellar (2006) descreve como a UK Water Industry Research Ltd (UKWIR) criou uma base de dados sobre falhas em adutoras. Esse autor relata que o ferro fundido foi o material que apresentou mais falhas com 29f/100km/ano, seguido do cimento-amianto com 17f/100Km/ano, PVC com 9f/100Km/ano, ferro dúctil com 7f/100Km/ano e PE com 4f/100Km/ano.

\section{METODOLOGIA E ESTUDO DE CASO}

A adutora de água tratada deste estudo de caso com 2735,1 m de comprimento está localizada em Campo Limpo Paulista e foi executada na 
década de 1970 com tubos de cimento-amianto DN $125 \mathrm{~mm}$. Desde sua implantação até a data da substituição, houve acréscimo da demanda de água em função do aumento da população abastecida. A adutora renovada é composta por $201 \mathrm{~m}$ de tubulação de ferro fundido DN 200 $\mathrm{mm}, 325 \mathrm{~m}$ de tubulação de ferro fundido $\mathrm{DN}$ $300 \mathrm{~mm}$ e $2209 \mathrm{~m}$ de PVC DEFOFO DN 300 mm. O bombeamento é realizado por um único conjunto motor-bomba. A bomba de trabalho é uma bomba KSB ETA 80-40/2 1750 rpm com rotor de $330 \mathrm{~mm}$ e a bomba reserva tem as mesmas características da anterior, porém com rotor de 210 $\mathrm{mm}$. Para a avaliação da variação no consumo de energia para os anos logo após a renovação da adutora, considerou-se um volume total recalcado anual constante, levando em conta as $\mathbf{7 9 0}$ ligações abastecidas pelo reservatório superior e uma população de 3,66 habitantes/ligação. Desse modo, não foram usados consumos específi$\cos \mathrm{kWh} / \mathrm{m}^{3}$ para as comparações, mas simplesmente os consumos em $\mathrm{kWh}$.

A escolha da nova tubulação foi feita considerando substituição do material antigo para evitar frequentes rompimentos, previsão de au- mento de demanda e redução de consumo de energia. Os tubos de ferro fundido DN $200 \mathrm{~mm}$ foram aplicados no trecho inicial dentro da área da EEAT e em uma travessia rodoviária, de forma a minimizar as interferências com as estruturas existentes e permitir a execução. Com o aumento do diâmetro da nova adutora, inaugurada em fevereiro de 2012, o consumo mensal de energia elétrica teve redução de $23,8 \%$ (de uma média mensal anual de 16168,52 kWh para 12316,23 kWh), como pode ser observado na Fig. 1, na qual estão apresentados os valores dos consumos mensais padronizados de 2011 a 2013. Para a determinação do consumo mensal padronizado de um dado mês, primeiramente divide-se o consumo mensal pelo número de dias do mês. Obtém-se, então, o consumo mensal padronizado multiplicando este resultado por 30. Apesar da tendência clara de diminuição do consumo de energia de 2011 para 2013, na comparação entre os anos de 2012 e 2013 são observados crescimentos para os meses de abril, maio e junho. Isso pode ter ocorrido por causa de variações sazonais nas demandas, que dependem de fatores climáticos, como temperatura e taxa de umidade por exemplo.

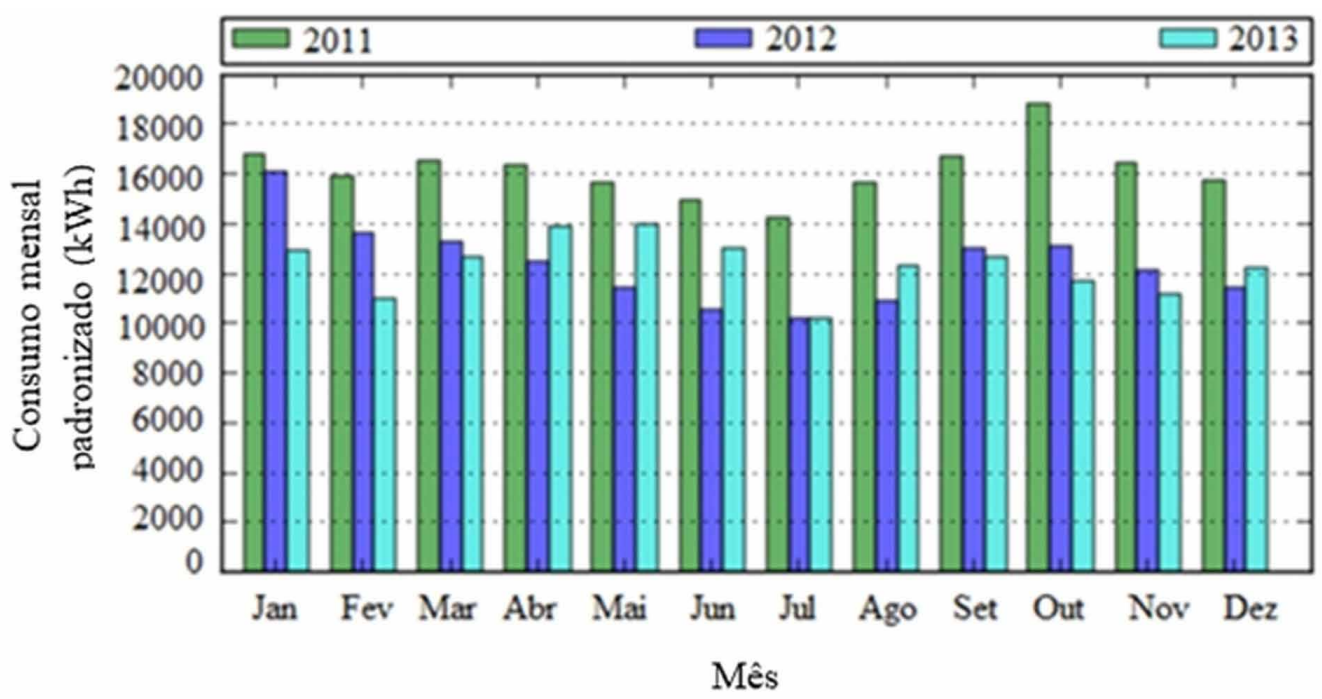

Figura 1: Redução do consumo de energia elétrica devido à substituição dos tubos da adutora. 
Com os dados conhecidos de consumo médio de energia elétrica e ponto de operação da bomba de vazão $69,7 \mathrm{~m}^{3} / \mathrm{h}\left(0,01936 \mathrm{~m}^{3} / \mathrm{h}\right)$ e rendimento de $71 \%$, foi determinada a operação do sistema de recalque inicial, em média $16,2 \mathrm{~h}$ por dia, resultando em um volume total recalcado de $1126,3 \mathrm{~m}^{3}$ por dia. A perda de carga devido ao atrito foi determinada pela equação de $\mathrm{Ha}$ zen-Williams, sendo $52 \mathrm{~m}$ para a situação antes da renovação e 0,64 $\mathrm{m}$ após. As perdas de carga localizadas foram desprezadas e as alturas manométricas $(\mathrm{Hm})$ calculadas para as situações inicial e final resultaram em $\mathrm{H}_{\mathrm{mi}}=82 \mathrm{~m} \mathrm{e}$ $\mathrm{H}_{\mathrm{mf}}=30,64 \mathrm{~m}$ respectivamente. 0 desnível geométrico existente no local é de $30 \mathrm{~m}$.
Foram determinados os custos do sistema adutor para a tubulação antiga, para a tubulação atual e para outros diâmetros de tubulação para fins de comparação. Considerou-se o preço dos tubos, custo de escavação e custo operacional. Considerou-se que o preço da tubulação de cimento-amianto DN $125 \mathrm{~mm}$, que não é mais produzida, é equivalente ao preço da tubulação de PVC DEFOFO de mesmo diâmetro. Por esse motivo, encontrou-se o preço unitário para PVC DEFOFO DN $125 \mathrm{~mm}$ por meio da interpolação com ajuste polinomial dos valores unitários de outros diâmetros conforme a Fig. 2. Os custos foram obtidos do banco de dados SINAPI e estão apresentados na Tabela 1.

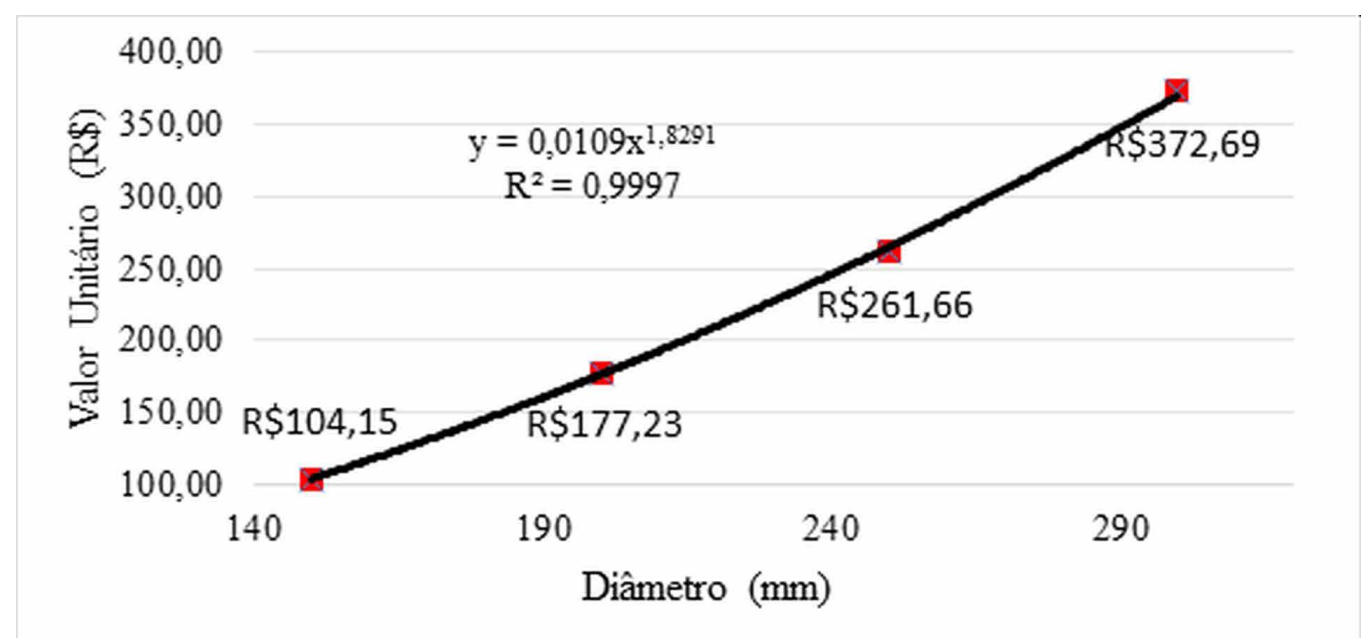

Figura 2: Curva de interpolação dos valores unitários dos diferentes diâmetros de tubulação de PVC DEFOFO.

Tabela 1: Custos calculados

\begin{tabular}{|c|c|}
\hline Tubulação & Custo \\
\hline Adutora antiga & $\mathrm{R} \$ 594.366,71$ \\
\hline Adutora renovada & $\mathrm{R} \$ 1.395 .244,57$ \\
\hline PVC DEFOFO DN $150 \mathrm{~mm}$ & $\mathrm{R} \$ 674.813,98$ \\
\hline PVC DEFOFO DN $200 \mathrm{~mm}$ & $\mathrm{R} \$ 883.771,95$ \\
\hline PVC DEFOFO DN $250 \mathrm{~mm}$ & $\mathrm{R} \$ 1.123 .519,75$ \\
\hline
\end{tabular}

A simples substituição da linha adutora por PVC DEFOFO DN 150 mm, sem considerar redução de consumo energético e aumento de demanda, teria um custo estimado de $\mathrm{R} \$ 674.813,98$. 0 custo estimado da tubulação atual é de $\mathrm{R} \$$ 1.395.244,57 e a economia de energia anual após a substituição da tubulação é de R\$19.281,70. É desejável, para a análise da viabilidade do aumento do diâmetro, levar em conta uma vida útil de 50 anos, segundo exigência da NBR7665 (ASSOCIAÇÃO BRASILEIRA DE NORMAS TÉCNICAS, 2007), bem como considerar que o aumento da vazão de recalque é função do crescimento vegetativo. $\mathrm{O}$ aumento do diâmetro pode ter efeito positivo em relação à prevenção de rompimentos e a adequação hidráulica com substituição do conjunto 
motor-bomba pode levar a uma economia maior, da ordem de $\mathrm{R} \$ 49.685,72$ ano.

Para o cálculo do consumo de energia teórico foram determinadas curvas características para cada tubulação considerada. As perdas de carga foram calculadas por meio da equação de HazenWilliams para algumas vazões de referência (Q $=20,40,60,80,100,120,140 \mathrm{~m}^{3} / \mathrm{h}$ ). As alturas manométricas foram determinadas somando a altura geométrica à perda de carga.

A partir dos dados de altura manométrica, foi possível escolher duas bombas adequadas para as tubulações. Adotou-se a bomba KSB 80-40/2 de 1740 rpm e diâmetro nominal do rotor de 330 mm para os cálculos com as tubulações de $125 \mathrm{e}$ 150 mm, e a bomba KSB 80-33 de 1710 rpm e diâmetro nominal do rotor de $310 \mathrm{~mm}$ para os cálculos com as tubulações de 200, 250 e 300 mm. O gráfico da Fig.3 apresenta os pontos de operação das bombas para diferentes diâmetros, pontos de interseção entre as curvas das bombas e as curvas das tubulações. O desnível geométrico de $30 \mathrm{~m}$ não foi alterado.

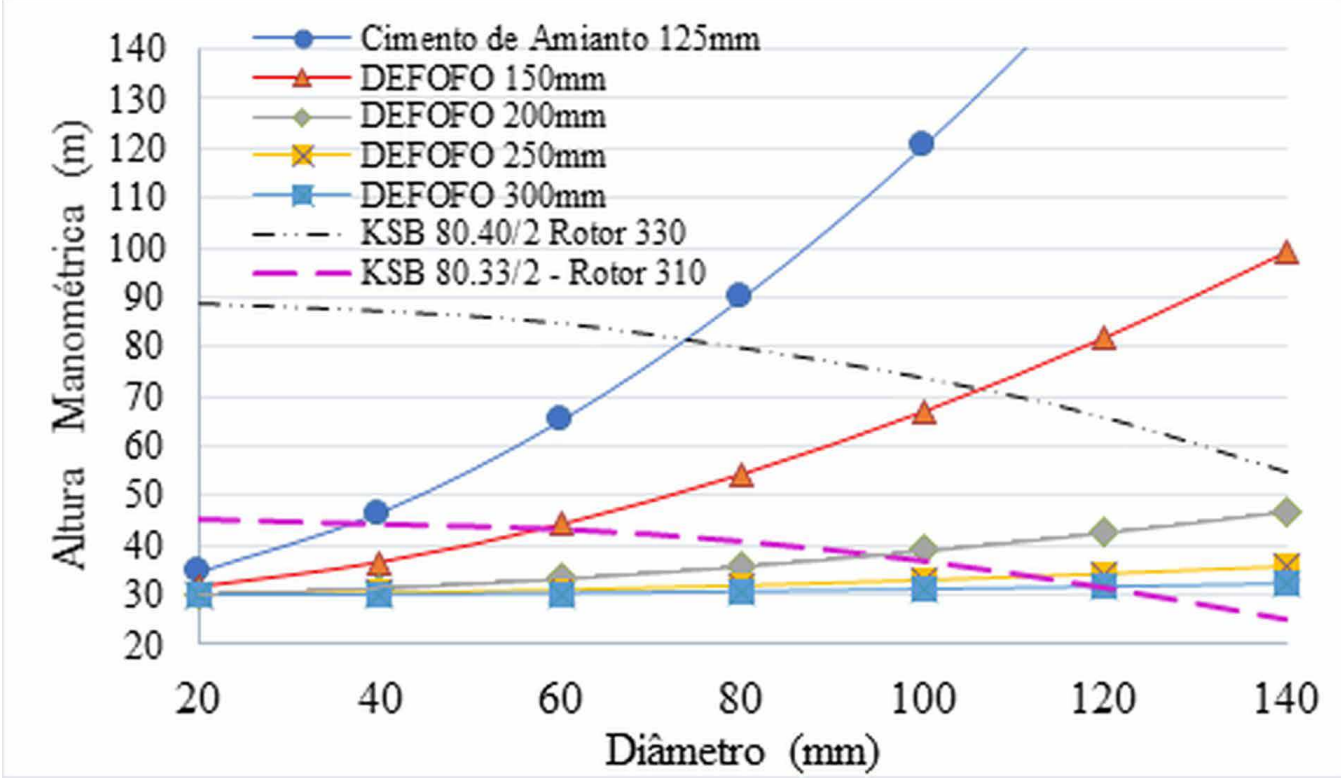

Figura 3: Gráfico para obtenção do ponto de operação das bombas para tubulações de diferentes diâmetros.

Por meio dos pontos obtidos pelo gráfico da Fig.3, foi possível encontrar os valores de potência para cada caso e os valores de consumo de energia para as diferentes tubulações. Adotou-se $94,5 \%$ para o rendimento do motor, de acordo com o que consta no site da fabricante WEG, fabricante do motor utilizado. 0 número de horas de funcionamento foi obtido pela divisão entre a vazão do ponto de operação e o volume de água diá- rio recalcado pelo sistema. O consumo mensal de energia elétrica foi obtido pela multiplicação da potência consumida pelo número de horas do mês (30 dias). A tarifa horária média de referência foi fornecida pela concessionária, no valor de $\mathrm{R} \$ 0,40331 / \mathrm{kWh}$. As Tabelas 2 e 3 apresentam os resultados encontrados para cada tubulação de acordo com a bomba considerada. 
Tabela 2: Cálculo da potência consumida e custo mensal do sistema adutor para diferentes diâmetros com a Bomba KSB ETA 80-40/2.

\begin{tabular}{|c|c|c|c|c|c|c|c|}
\hline \multicolumn{8}{|c|}{ KSB 80 - 40/2 - 1740 rpm - Rotor 330 mm } \\
\hline Diâmetro (mm) & $\begin{array}{c}\text { Vazão Ponto } \\
\text { Operação } \\
\left(\mathrm{m}^{3} / \mathrm{h}\right)\end{array}$ & $\begin{array}{c}H_{m} \text { Ponto } \\
\text { Operação }(m)\end{array}$ & $\begin{array}{c}\text { Horas de } \\
\text { funcionamento }\end{array}$ & $\begin{array}{c}\text { Rendimento da } \\
\text { bomba }\end{array}$ & $\begin{array}{c}\text { Potência } \\
\text { necessária (HP) }\end{array}$ & $\begin{array}{l}\text { Potência } \\
\text { Consumida } \\
\text { (kW) }\end{array}$ & Custo Mensal \\
\hline 125 & 69,7 & 82,9 & 16,2 & 0,71 & 30 & 33,34 & $\mathrm{R} \$ 6.519,24$ \\
\hline 150 & 105,8 & 71,5 & 10,6 & 0,75 & 38 & 39,98 & $\mathrm{R} \$ 5.149,95$ \\
\hline
\end{tabular}

Tabela 3: Cálculo da potência consumida e custo mensal do sistema adutor para diferentes diâmetros com a Bomba KSB ETA 80-33.

\begin{tabular}{|c|c|c|c|c|c|c|c|}
\hline \multicolumn{8}{|c|}{ Bomba KSB 80 - 33 - 1710 rpm - Rotor 310 mm } \\
\hline Diâmetro (mm) & $\begin{array}{c}\text { Vazão Ponto } \\
\text { Operação } \\
\left(\mathrm{m}^{3} / \mathrm{h}\right)\end{array}$ & $\begin{array}{c}H_{m} \text { Ponto } \\
\text { Operação }(m)\end{array}$ & $\begin{array}{c}\text { Horas de } \\
\text { Funcionamento }\end{array}$ & $\begin{array}{c}\text { Rendimento da } \\
\text { Bomba }\end{array}$ & $\begin{array}{c}\text { Potência } \\
\text { Necessária (HP) }\end{array}$ & $\begin{array}{l}\text { Potência } \\
\text { Consumida } \\
\text { (kW) }\end{array}$ & Custo Mensal \\
\hline 200 & 92,9 & 38,16 & 12,1 & 0,73 & 18 & 19,46 & $\mathrm{R} \$ 2.640,53$ \\
\hline 250 & 110,2 & 34,02 & 10,2 & 0,70 & 19 & 21,42 & $\mathrm{R} \$ 2.349,67$ \\
\hline 300 & 114,5 & 32,8 & 9,8 & 0,69 & 20 & 22,87 & $\mathrm{R} \$ 2.380,45$ \\
\hline
\end{tabular}

O consumo de energia para o diâmetro de $250 \mathrm{~mm}$ é menor do que para o diâmetro de $300 \mathrm{~mm}$. Isso ocorre porque a redução da perda de carga com o aumen- to da tubulação de $250 \mathrm{~mm}$ para $300 \mathrm{~mm}$ não é tão significativa e a bomba apresenta melhor rendimento para o ponto de operação do diâmetro de $250 \mathrm{~mm}$.

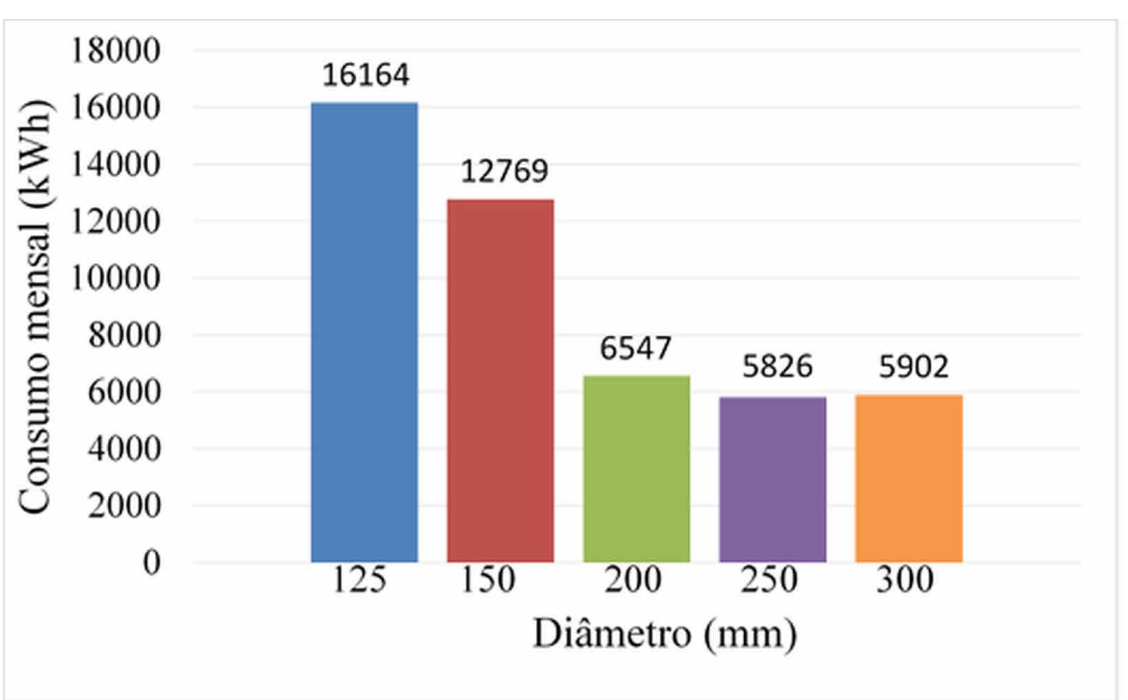

Figura 4: Gráfico do consumo de energia elétrica mensal teórica para diferentes diâmetros (material DeFOFO, exceto para $\mathrm{DN}=125 \mathrm{~mm}$ com cimento-amianto)

O consumo de energia mensal encontrado para a tubulação de cimento-amianto DN 125 mm (obtido com base no estudo teórico) é semelhante ao consumo mensal médio real fornecido pela concessionária. Já o consumo energético men- sal encontrado para a tubulação de PVC DEFOFO com diâmetro de $300 \mathrm{~mm}$, de 6.272,22 kWh, é diferente da média de consumo do estudo de caso após a substituição da tubulação da adutora, de 12.178,77 kWh. Essa diferença pode ser 
oriunda de restrições hidráulicas na operação do sistema de adução, que pode estar operando com baixo rendimento, e devido à consideração de outra bomba (a KSB ETA 80-33) para o cálculo do consumo teórico. A Fig.4 apresenta o consumo teórico mensal para cada diâmetro.

Considerando a redução de consumo de energia com base no cálculo teórico, para a linha adutora DN $300 \mathrm{~mm}$, e comparando com a adutora antiga de $125 \mathrm{~mm}$, a redução de consumo de energia seria da ordem de 10.266,24 kW mensais, representando uma redução de $63,4 \%$ no custo de operação com energia elétrica.

\section{RESULTADOS E DISCUSSÃO}

\subsection{Diâmetro econômico teórico}

A partir dos resultados obtidos para o custo da tubulação e para o consumo de energia elétrica teóricos, obtém-se o gráfico da Fig.5 para a determinação do diâmetro econômico para a tubulação do estudo de caso. Considerou-se o custo total anual da tubulação como sendo o valor total da obra para cada diâmetro dividido por 30 anos, tempo comum de concessão dos sistemas de abastecimento de água. Devido ao longo período de 30 anos, o custo do conjunto motor-bomba não foi considerado. Por meio do gráfico, obtém-se o diâmetro econômico de $210 \mathrm{~mm}$.

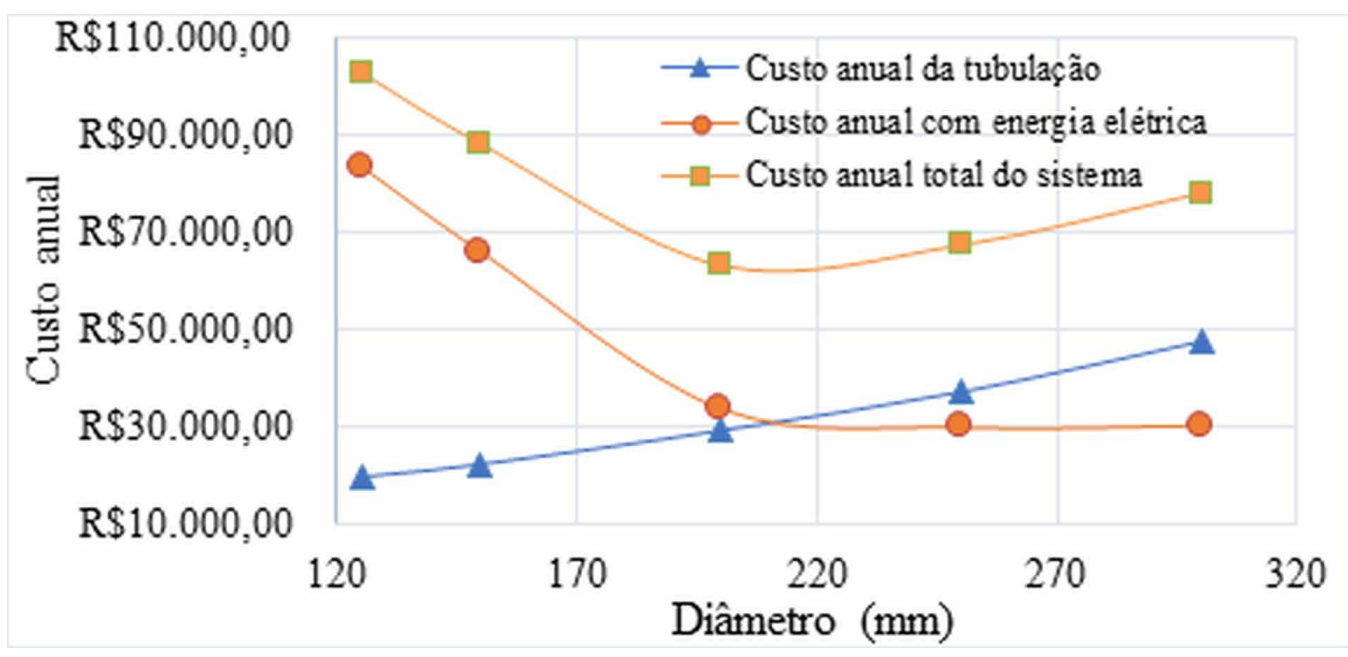

Figura 5: Determinação do diâmetro econômico de uma tubulação de recalque.

A partir dos custos teóricos de energia elétrica mensal, apresentados nas Tabelas 2 e 3, para as tubulações de cimento-amianto e PVC DEFOFO $200 \mathrm{~mm}$, pode-se obter a diferença anual para operação de $\mathrm{R} \$ 46.544,49$. A diferença de custo total das tubulações é de $\mathrm{R} \$ 289.405,24$. A adoção do diâmetro de $200 \mathrm{~mm}$ poderia levar a uma economia no custo de energia elétrica com retorno do investimento satisfatório, que depende evidentemente da análise que envolve o custo do capital investido e o valor e a previsão de reajuste anual da tarifa de energia elétrica. $O$ custo de energia elétri- ca no Brasil, importante parâmetro na análise para a definição do diâmetro, pode variar bastante ao longo dos anos, devido a condições econômicas ou de produção de energia instáveis.

\subsection{Diâmetro econômico pela fórmula de Bresse}

Para o cálculo do diâmetro econômico por Bresse, considera-se a fórmula universal de Darcy-Weisbach para perda de carga distribuída. Encontram-se valores de f próximos para as tubulações 
estudadas. Por esse motivo, optou-se por utilizar o valor médio de $f=0,019$ para o cálculo do diâmetro econômico pela fórmula de Bresse. A vazão considerada foi de $0,0195 \mathrm{~m}^{3} / \mathrm{s}$.

Para o rendimento do motor $\eta \mathrm{m}$, adotou-se $94,5 \%$. O rendimento da bomba $\eta$ varia com a vazão e a altura manométrica. Adotou-se rendimento de $70 \%$, resultando em um rendimento total de $66,15 \%$.
Calculou-se o custo de $1 \mathrm{~m}$ de conduto com $1 \mathrm{~m}$ de diâmetro de tubulação de PVC DEFOFO por meio de interpolação com ajuste linear dos valores para diferentes diâmetros, obtendo-se R $\$ 1608,51$. Considerou-se custo de manutenção de $2 \%$ do custo da tubulação ao ano, obtendo-se, então, o valor de p1 da Equação de Bresse conforme a Eq. 12. Para a taxa de amortização do capital, considerou-se 10\% ao ano, comum a investimentos deste porte.

$p_{1}=1608,50 \times 1,10+32,17=R \$ 1801,53$

Considerou-se, como custo de operação, o valor médio das tarifas fornecido pela concessionária multiplicado pelo consumo anual conforme a Eq. 13.

$p_{2}=163.409,46 \mathrm{kWh} \times 0,40331 R \$ / k W h+c_{m}$

Para o cálculo do custo de manutenção $(\mathrm{cm})$, considerou-se um valor de $R \$ 30.000,00$ com vida útil de 10 anos. Assim, considerou-se o custo de manutenção como sendo R $\$ 3000.00$ por ano conforme a Eq. 14 .

$$
p_{2}=163.409,46 k W h \times 0,40331 \frac{R \$}{k W h}+R \$ 3.000,00=R \$ 68.904,67
$$

O diâmetro econômico encontrado pela Fórmula de Bresse foi de $179 \mathrm{~mm}$. Adotando-se o diâmetro nominal superior mais próximo, tem-se 200 $\mathrm{mm}$. Comparando-se com o resultado do dimensionamento econômico, obtido a partir do cálculo teórico do consumo mensal de energia elétrica para tubulações de diferentes diâmetros para os dados do estudo de caso, pode-se concluir que a fórmula de Bresse apresentou um resultado satisfatório que, aproximado para o diâmetro comercial mais próximo, fornece diâmetro semelhante ao obtido na análise teórica, $210 \mathrm{~mm}$. Pode-se calcular também, pela Eq.4, o valor do coeficiente de Bresse $K=1,28$, um valor considerado razoável conforme indicam vários autores.

\subsection{Diâmetro econômico pelo método de Prevedello}

Calculou-se o diâmetro econômico pelo método de Prevedello, conforme a Eq.11. Para resolver essa equação, faz-se uso de processo iterativo. Para esse fim, o software MATLAB foi usado. Como etapa prévia, os parâmetros de entrada foram determinados.

Determinou-se a por meio da análise do gráfico do preço unitário versus diâmetro para os tubos de PVC DEFOFO com diâmetros de 150, 200, 250 e $300 \mathrm{~mm}$. Encontrou-se $\alpha=1,8291$, conforme Fig. 2. 
Também por meio da análise gráfica, determinou-se o custo da canalização $p$ por $\mathrm{cm}$ de diâmetro e $\mathrm{m}$ de comprimento. Encontrou-se $\mathrm{p}=$ $\mathrm{R} \$ 7,4 / \mathrm{cm} / \mathrm{m}$. Para a tubulação de PVC DEFOFO, adotou-se rugosidade absoluta $\mathrm{k}=0,01 \mathrm{~mm}$, conforme Porto (2006). Considerou-se rendimento do conjunto motor-bomba de $66,15 \%$. Foram utilizados os seguintes dados adicionais para resolução: $\gamma=997,05 \mathrm{kgF} / \mathrm{m}^{3}, \mathrm{Q}=0,0195$ $\mathrm{m}^{3} / \mathrm{s}, \mathrm{n}=16 \mathrm{~h}, \mathrm{~s}=0,40331 \mathrm{R} \$ / \mathrm{h}, \mathrm{r}=10 \%$ a.a., $\nu=$ $0,000001 \mathrm{~m}^{2} / \mathrm{s}$.

Obteve-se o diâmetro D = 194 mm. Arredondando-se para o diâmetro comercial mais próximo, o diâmetro econômico obtido pelo método de Prevedello é de $200 \mathrm{~mm}$.

\section{CONCLUSÕES}

Neste trabalho foi estudada a renovação de uma adutora por recalque localizada em Campo Limpo Paulista, interior do estado de São Paulo. A adutora antiga de cimento-amianto $(125 \mathrm{~mm})$ foi substituída pela concessionária por outra com tubos de PVC DEFOFO com maior diâmetro (300 mm), com o objetivo de economizar energia e aumentar os níveis de segurança. Nesse contexto, determinou-se o diâmetro econômico por meio de uma análise que usou alguns dados disponibilizados pela concessionária e por meio de formulações da literatura (fórmula de Bresse e método de Prevedello).

A partir dos dados do estudo de caso, dos orçamentos para instalação de tubulações com diferentes diâmetros e do cálculo teórico do consumo de energia elétrica do sistema de recalque, pode-se calcular o diâmetro econômico para a tubulação do estudo de caso, que é o diâmetro da tubulação que gera o menor custo anual global do sistema. Foi obtido o diâmetro teórico de $210 \mathrm{~mm}$.
A fórmula de Bresse conduziu a um diâmetro de $179 \mathrm{~mm}$, um resultado que indica a adoção do diâmetro nominal de 200 mm, próximo do valor encontrado pela análise teórica. 0 método de Prevedello, considerando $16 \mathrm{~h}$ de funcionamento diário, conduziu a um diâmetro de $194 \mathrm{~mm}$, valor superior ao obtido em relação ao método de Bresse. Apesar das diferenças entre as abordagens de Bresse e Prevedello quanto à consideração da quantidade de horas de funcionamento diário, considerou-se a mesma vazão de $0,0195 \mathrm{~m}^{3} / \mathrm{s}$.

Tem-se, portanto, que as metodologias usadas para obtenção do diâmetro otimizado fornecem resultados próximos. A substituição da adutora deste estudo de caso gerou uma redução de custos de operação com energia elétrica da ordem de $23,8 \%$ entre os anos de 2011 e 2013. Com ajustes hidráulicos e de equipamentos, de acordo com o estudo teórico realizado, a economia pode chegar a $63,4 \%$.

\section{AGRADECIMENTOS}

Agradecemos à Companhia de Saneamento Básico do Estado de São Paulo (Sabesp), pela disponibilização de dados. Agradecemos também à Coordenação de Aperfeiçoamento de Pessoal de Nível Superior (Capes) e ao Conselho Nacional de Desenvolvimento Científico e Tecnológico (CNPq) pelo apoio financeiro para a realização desta pesquisa. Agradecemos também à Fundação de Amparo à Pesquisa do Estado de São Paulo FAPESP, processo 2017/09921-2 pelo apoio financeiro.

\section{CONTRIBUIÇÃO DOS AUTORES}

Todos os autores contribuíram de forma igualitária. 


\section{REFERÊNCIAS}

ASSOCIAÇÃO BRASILEIRA DE NORMAS TÉCNICAS. NBR 7665 Sistemas para adução e distribuição de água - Tubos de PVC DEFOFO com junta elástica. Rio de Janeiro, 2007.

BOWEN, J.; DAVIES, L.; BURDETT, G. J.; BARBER, C. M. Managing Asbestos-Containing Materials in the Built Environment: Report of a Health and Safety Executive and Government Office for Science Workshop. Annals of Work Exposures and Health, v. 61, n. 1, p. 16-21, 2017. https://doi.org/10.1093/annweh/wxw007

IARC. Asbestos (chrysotile, amosite, crocidolite, tremolite, actinolite, and anthophyllite). IARC Monographs on the Evaluation of Carcinogenic Risks to Humans - 100 C, 2012.

MACKELLAR, S. UKWIR National Mains Failure Database. International Conference of Plastic Pipes XIII, 2006. Anais...

MAGNANI, J. R. Determinação do diâmetro econômico de adutoras e emissários por recalque. Revista DAE, v. 113, n. 259, p. 13, 1968.

PLAPPALLY, A. K.; LIENHARD V, J. H. Energy requirements for water production, treatment, end use, reclamation, and disposal. Renewable and Sustainable Energy Reviews, v. 16, n. 7, p. 48184848, 2012. https://doi.org/10.1016/j.rser.2012.05.022

PORTO, R. M. Hidráulica Básica. $4^{\mathrm{a}}$ Edição. Escola de Engenharia de São Carlos (Universidade de São Paulo), 2006.
PREVEDELLO, C. L. Diâmetro mais econômico de uma canalização de recalque. Revista Brasileira de Recursos Hídricos, v. 5, n. 4, p. 39-42, 2000. https://doi.org/10.21168/rbrh.v5n2.p39-42

RAMOS, H. M.; KENOV, K. N.; VIEIRA, F. Environmentally friendly hybrid solutions to improve the energy and hydraulic efficiency in water supply systems. Energy for Sustainable Development, v. 15, n. 4, p. 436-442, 2011. https://doi.org/10.1016/j.esd.2011.07.009

RICHTER, C. A. Diâmetro econômico das canalizações de recalque (adaptação e atualização das fórmulas de Koch e Vibert). Revista DAE, v. 133, n. 1130, p. 2, 1983.

ROBAINA, A. D.; CALGARO, M.; PIETER, M. X. Tubulações em sistemas de recalque. Ciência Rural, p. 1065-1068, 2004.

TELES, E. G. Novo processo para a determinação do diâmetro mais econômico para adutoras de recalque. Revista DAE, v. 65, n. 913, 1967.

TULLIS, J. P. Hydraulic of pipelines, pumps, valves, cavitation, transients. Nova York: John Wiley \& Sons, 1989.

VILANOVA, M. R. N.; BALESTIERI, J. A. P. Energy and hydraulic efficiency in conventional water supply systems. Renewable and Sustainable Energy Reviews, v. 30, p. 701-714, 2014. https://doi. org/10.1016/j.rser.2013.11.024 